\title{
Age-dependent effects of cord blood long-chain PUFA composition on BMI during the first 10 years of life
}

\author{
Marie Standl ${ }^{1 *}$, Elisabeth Thiering ${ }^{1,2}$, Hans Demmelmair ${ }^{2}$, Berthold Koletzko ${ }^{2}$ and Joachim Heinrich ${ }^{1}$ \\ ${ }^{1}$ Institute of Epidemiology I, Helmboltz Zentrum München - German Research Centre for Environmental Health, \\ Ingolstädter Landstraße 1, Neuberberg 85764, Germany \\ ${ }^{2}$ University of Munich Medical Centre, Dr von Hauner Children's Hospital, Munich, Germany
}

(Submitted 12 August 2013 - Final revision received 19 December 2013 - Accepted 10 January 2014 - First published online 13 February 2014)

\begin{abstract}
In the present study, we investigated whether $n-6$ and $n-3$ long-chain PUFA (LC-PUFA) concentrations in cord blood (CB) serum are associated with BMI up to 10 years of age, after accounting for LC-PUFA composition at 2, 6 and 10 years. The study was based on 388 participants of the German LISAplus (Influence of Lifestyle-Related Factors on the Immune System and the Development of Allergies in Childhood Plus the Influence of Traffic Emissions and Genetics) birth cohort study carried out in Munich. BMI was measured at 2, 6 and 10 years of age. Serum phospholipid fatty acid concentrations were measured by GC in CB and in blood collected at 2,6 and 10 years of age. The association between $n$-3 LC-PUFA and $n$-6 LC-PUFA concentrations and $n$-6:n-3 LC-PUFA ratio in CB serum glycerophospholipids and BMI $z$-scores was assessed using linear mixed models adjusted for LC-PUFA composition at follow-up and potential confounders. Interaction terms between time of follow-up and LC-PUFA concentrations in CB were included. There was no consistent association between $n-6$ and $n-3$ LC-PUFA concentrations in CB and BMI over time. However, there was a significant interaction between $n-6: n-3$ LC-PUFA ratio in CB and time of follow-up with respect to BMI $(P=0.0415)$ : a negative effect at 2 years; no effect at 6 years; a positive effect at 10 years. BMI up to 10 years of age may be influenced by the $n-6: n-3$ ratio in CB serum glycerophospholipids in a time-varying fashion. The present results thereby highlight the importance of considering age when examining associations between fatty acid concentrations and BMI.
\end{abstract}

\section{Key words: BMI: Children: PUFA: Epidemiology: Cord blood}

The prevalence of overweight and obesity in children has increased over the past few decades. Early-life obesity is an emerging risk factor for obesity and obesity-related diseases in adulthood ${ }^{(1-3)}$. In addition to other factors, altered fatty acid composition in diet, especially an elevated ratio of $n$-6:n-3 long-chain PUFA (LC-PUFA), has been suggested to be a potential contributing factor ${ }^{(4,5)}$.

Adipocyte development and fat deposition in the fetus increase exponentially with gestational age ${ }^{(6)}$. During the last trimester of gestation, LC-PUFA accumulate in the placenta $^{(6,7)}$, leading to higher concentrations of LC-PUFA in cord blood (CB) than in maternal plasma. CB LC-PUFA concentrations are associated with maternal dietary intake and maternal LC-PUFA concentrations in plasma ${ }^{(8,9)}$.

Postnatally, the increase in the number and size of adipocytes is highest during the first year of life, although the differentiation of precursor cells into adipocytes also continues later in life $e^{(5,7)}$. Thus, prenatal and early postnatal periods are critical for adipose tissue development.
Ailhaud et al. ${ }^{(5,10)}$ proposed that the qualitative changes in ingested fats, specifically the increased intake of $n-6$ PUFA, which yields a higher $n-6: n-3$ ratio, may promote adipose tissue development. Arachidonic acid (AA), the main n-6 PUFA metabolite, is a precursor of eicosanoids, such as prostacyclin. Prostacyclin enhances the differentiation of adipose precursor cells into adipocytes. By contrast, the $n$ - 3 LC-PUFA EPA and DHA attenuate the production of eicosanoids and, therefore, are less adipogenic and inhibit adipose tissue development ${ }^{(5,10)}$.

So far, despite a biologically plausible mechanism, evidence for an association between CB LC-PUFA composition and adipose tissue development is scarce. In 2010, a Cochrane review of interventional studies on the effect of LC-PUFA supplementation in breast-feeding mothers on child growth has led to a conclusion of insufficient evidence for this association ${ }^{(11)}$. No association between LC-PUFA supplementation and weight has been found, but a negative association between LC-PUFA supplementation and child length has been found.

Abbreviations: AA, arachidonic acid; CB, cord blood; LC-PUFA, long-chain PUFA; zBMI, BMI $z$-scores.

*Corresponding author: M. Standl, fax + 4989 31873380, email marie.standl@helmholtz-muenchen.de 
A more recently published review has also led to inconclusive results $^{(12)}$, despite two of the reviewed interventional studies reporting a modest increase in birth weight associated with maternal $n$-3 LC-PUFA supplementation. The INFAT (impact of nutritional fatty acids during pregnancy and lactation on early adipose tissue development) study, which investigated the effect of $n-3$ fatty acid supplementation during pregnancy and lactation period on adipose tissue growth during the first year of life, found no effects. However, this study was limited by sample size, as only approximately 100 subjects were included in each group ${ }^{(13)}$. This sample size was required based on a calculation with an $\alpha$-level of 5 and $80 \%$ power to detect differences of at least $5 \mathrm{~mm}$ in the sum of four defined skinfolds between the intervention and control groups at 4 months ${ }^{(14)}$.

In the present study, we aimed to investigate how the concentrations of $n$ - 3 LC-PUFA and $n$ - 6 LC-PUFA and the ratio of $n-6: n-3$ LC-PUFA in CB serum glycerophospholipids affect BMI later in life using a longitudinal study design based on repeated BMI and LC-PUFA measurements. The concentrations of $n$ - 3 LC-PUFA and $n$ - 6 LC-PUFA and the ratio of $n$-6:n-3 LC-PUFA at follow-up examinations later in life were included in the analysis to rule out confounding by lifestyle or dietary factors, which may be reflected in the fatty acid composition in blood. We hypothesise that higher $n-3$ LC-PUFA concentrations, lower $n-6$ LC-PUFA concentrations and a lower $n$-6:n-3 LC-PUFA ratio in CB serum glycerophospholipids are associated with a lower BMI up to 10 years of age, even after accounting for serum glycerophospholipid LC-PUFA concentrations later in life.

\section{Subjects and methods \\ Study population}

The German LISAplus (Influence of Lifestyle-Related Factors on the Immune System and the Development of Allergies in Childhood Plus the Influence of Traffic Emissions and Genetics) is a population-based birth cohort study in which a total of 3097 neonates were recruited between 1997 and 1999 from the German cities of Munich, Leipzig, Wesel and Bad Honnef. Details of the study design have been described elsewhere $^{(15)}$. During recruitment in maternity wards, CB samples were collected and deep-frozen until the time of measurement. Questionnaires were completed by the parents at birth and $0 \cdot 5,1,1 \cdot 5,2,4,6$ and 10 years of age, and physical examinations took place at 2, 6 and 10 years. During the physical examinations, blood samples were collected and height and weight were measured.

Participant flow is shown in Fig. 1. The substudy was restricted to children from the Munich study centre. Of the 1467 successfully recruited children, CB samples could be collected from 814. These $814 \mathrm{CB}$ samples were used to measure total IgE concentrations ${ }^{(15)}$, and a sufficient amount of serum collected from 681 children remained for the measurement of fatty acid composition. Fatty acid composition was measured in these remaining 681 CB samples.

Approval from the local ethics committees (Bavarian Board of Physicians, University of Leipzig, Board of Physicians

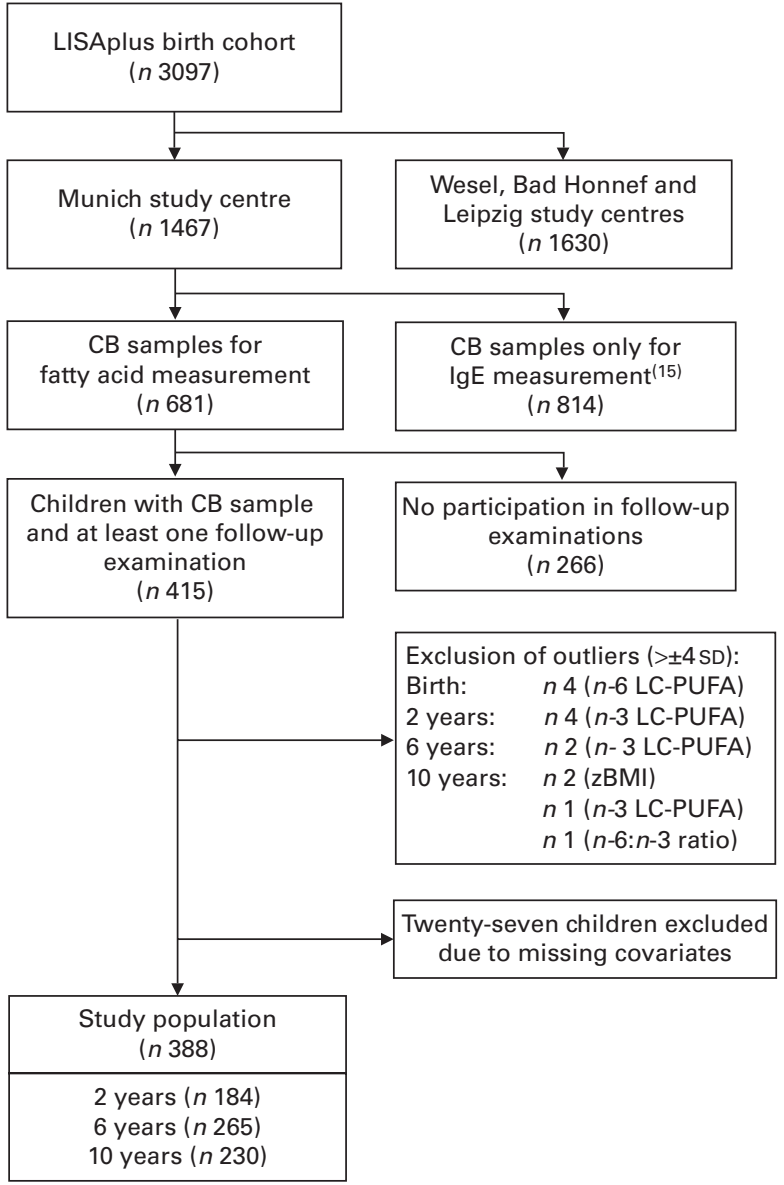

Fig. 1. Participant flow. LISAplus birth cohort, Influence of Lifestyle-Related Factors on the Immune System and the Development of Allergies in Childhood Plus the Influence of Traffic Emissions and Genetics birth cohort; $C B$, cord blood; LC-PUFA, long-chain-PUFA; zBMI, BMI z-scores.

of North Rhine-Westphalia) and written consent from the participants' families were obtained.

\section{Calculation of $B M / z$-scores}

BMI was calculated during each follow-up at 2, 6 and 10 years of age and transformed to age- and sex-specific standard deviation scores ( $z$-scores) according to the WHO growth standards ${ }^{(16)}$.

\section{Measurement of fatty acid concentrations}

Fatty acid analysis was carried out by selective transfer of glycerophospholipid fatty acids from $100 \mu \mathrm{l}$ serum into their methyl esters and their gas chromatographic separation and quantification $^{(17)}$. This procedure has been described previously in detail for the analysis of serum from $\mathrm{CB}$ and from blood samples collected at the ages of 2 and 6 years ${ }^{(18,19)}$ and has also been applied for blood samples collected at the age of 10 years.

Total $n-6$ LC-PUFA concentration was calculated by summing up eicosadienoic acid $(20: 2 n-6)$, dihomo- $\gamma$-linolenic acid $(20: 3 n-6)$, AA $(20: 4 n-6)$, adrenic acid $(22: 4 n-6)$ and 
docosapentaenoic acid $(22: 5 n-6)$ concentrations. Total $n$-3 LC-PUFA concentration was calculated by summing up eicosatrienoic acid (20:3n-3), EPA (20:5n-3), docosapentaenoic acid (22:5n-3) and DHA (22:6n-3) concentrations. Total $n-6$ LC-PUFA and total $n$ - 3 LC-PUFA concentrations are presented as a percentage of the concentrations of all the measured fatty acids with fourteen to twenty-four carbon atoms. The $n-6: n-3$ LC-PUFA ratio was calculated by dividing total $n-6$ LC-PUFA concentrations by total $n-3$ LC-PUFA concentrations.

\section{Statistical analyses}

Children with BMI $z$-scores (zBMI) or fatty acid concentrations deviating more than \pm 4 SD from the mean were considered as outliers and treated as missing values in the respective analysis (Fig. 1). This affected at birth four children for n-6 LC-PUFA concentrations, at 2 years four children for $n$-3 LC-PUFA concentrations, at 6 years two children for $n$ - 3 LC-PUFA concentrations, and at 10 years two children for zBMI, one child for $n$-3 LC-PUFA concentrations and one child for $n-6: n-3$ LC-PUFA ratio. All the investigated variables were tested for differences between study participants and non-participants using Fisher's exact test for binary variables, Wilcoxon ranksum test for continuous, not normally distributed variables and two-sample $t$ test for normally distributed variables.

Correlations between repeated measurements of zBMI, $n-6$ LC-PUFA concentrations, $n-3$ LC-PUFA concentrations and $n-6: n-3$ LC-PUFA ratio were determined and tested using Pearson's correlation.

Linear mixed models, which are an extension of linear regression to longitudinal data, were applied to account for the repeated $z B M I$ measurements ${ }^{(20)}$. A random intercept was included in the model for each subject, which modelled the change per individual over time. As such, the same number of measurements per subject was not required. Inclusion criteria for this analysis were the availability of information on CB LC-PUFA composition and on zBMI and LC-PUFA measurements for at least one of the follow-up time points (2, 6 or 10 years). The model included variables that were the same for each time point (LC-PUFA composition in $\mathrm{CB}$, birth weight, maternal BMI before pregnancy (subjects with a BMI $>46 \mathrm{~kg} / \mathrm{m}^{2}$ were excluded) and exclusive breastfeeding (defined as a binary variable: exclusive breast-feeding for at least 4 months $v$. no exclusive breast-feeding or exclusive breast-feeding for $<4$ months)) as well as time-varying variables (age, time of follow-up and LC-PUFA composition at 2, 6 and 10 years). The linear mixed models returned one estimate for the variables that were measured once (CB LC-PUFA composition) and one combined estimate for the variables that were measured at the various time points (LC-PUFA composition at follow-up).

Based on the results of initial explanatory analyses, three interaction terms were included in the model (between time of follow-up and LC-PUFA composition in CB, LC-PUFA concentrations at the time of follow-up and maternal BMI before pregnancy). Additionally, maternal age at birth, maternal education level (low: less than tenth grade; medium: tenth grade; and high: more than tenth grade), maternal smoking during pregnancy, parity (defined as number of pregnancies; coded as 0 if the child under study was firstborn and 1 otherwise) and sex were tested as covariables, but these did not exhibit a significant association in the fully adjusted model and were therefore not included in the final model. Statistical significance was defined by a two-sided $\alpha$-level of $5 \%$.

Additionally, linear regression was conducted for each follow-up, regressing LC-PUFA composition in $\mathrm{CB}$ on zBMI adjusted for LC-PUFA composition at the time of follow-up, age, birth weight, maternal BMI before pregnancy and exclusive breast-feeding for at least 4 months.

The linear mixed model does not require that each subject have the same number of data points. To rule out a potential bias that may be introduced by non-random loss to follow-up,

Table 1. Characteristics of the study population

(Mean values and standard deviations or percentages)

\begin{tabular}{|c|c|c|c|c|c|c|c|c|}
\hline & \multicolumn{2}{|c|}{$\begin{array}{c}\text { CB } \\
(n 388)\end{array}$} & \multicolumn{2}{|c|}{$\begin{array}{l}2 \text { years } \\
(n 184)\end{array}$} & \multicolumn{2}{|c|}{$\begin{array}{l}6 \text { years } \\
(n 265)\end{array}$} & \multicolumn{2}{|c|}{$\begin{array}{c}10 \text { years } \\
(n 230)\end{array}$} \\
\hline & Mean & SD & Mean & SD & Mean & SD & Mean & SD \\
\hline Age (years) & & & $2 \cdot 0$ & 0.0 & $6 \cdot 1$ & 0.2 & $10 \cdot 2$ & $0 \cdot 2$ \\
\hline zBMI & & & 0.2 & $1 \cdot 0$ & 0.0 & 0.90 & 0.0 & 1.0 \\
\hline$n-6$ LC-PUFA $(\%)^{*}$ & $25 \cdot 3$ & 1.5 & $13 \cdot 4$ & 1.5 & $13 \cdot 7$ & 1.6 & $15 \cdot 0$ & 1.6 \\
\hline$n-3$ LC-PUFA (\%)* & $8 \cdot 1$ & 1.6 & 4.5 & 0.8 & 4.5 & 0.9 & $4 \cdot 8$ & $1 \cdot 0$ \\
\hline$n-6: n-3$ LC-PUFA ratio* & $3 \cdot 2$ & 0.7 & $3 \cdot 0$ & 0.5 & $3 \cdot 1$ & 0.6 & $3 \cdot 2$ & 0.6 \\
\hline Sex (female) (\%) & \multicolumn{2}{|c|}{43.6} & & & & & & \\
\hline Maternal BMI $\left(\mathrm{kg} / \mathrm{m}^{2}\right)$ & $22 \cdot 2$ & 3.4 & & & & & & \\
\hline Birth weight $(\mathrm{g})$ & $3429 \cdot 3$ & $391 \cdot 1$ & & & & & & \\
\hline Exclusive breast-feeding for $>4$ months (\%) & \multicolumn{2}{|c|}{49.5} & & & & & & \\
\hline Maternal age (years) & $32 \cdot 8$ & $4 \cdot 0$ & & & & & & \\
\hline Smoking during pregnancy (\%) & \multicolumn{2}{|c|}{11.6} & & & & & & \\
\hline \multicolumn{9}{|l|}{ Maternal education level (\%) } \\
\hline Low & \multicolumn{2}{|c|}{$7 \cdot 2$} & & & & & & \\
\hline Medium & \multicolumn{2}{|c|}{$27 \cdot 1$} & & & & & & \\
\hline High & \multicolumn{2}{|c|}{$65 \cdot 7$} & & & & & & \\
\hline Parity (\%; firstborn) & \multicolumn{2}{|c|}{$50 \cdot 1$} & & & & & & \\
\hline
\end{tabular}

CB, cord blood; zBMI, BMI z-scores; LC-PUFA, long-chain-PUFA.

${ }^{\star}$ Measured in serum glycerophospholipids. 
Table 2. Pearson's correlation between time of follow-up and BMI z-scores (zBMI)

\begin{tabular}{lccc}
\hline zBMI & 2 years & 6 years & 10 years \\
\hline 2 years & 1 & & \\
6 years & $0.52^{\star * *}$ & 1 & \\
10 years & $0.20^{\star}$ & $0.75^{\star \star *}$ & 1 \\
\hline${ }^{*} P<0.05,{ }^{* * *} P<0.001$. & &
\end{tabular}

an additional sensitivity analysis was conducted. This analysis was restricted to children with complete information on zBMI and fatty acid measurements at all time points.

Statistical analyses were carried out using R, version 2.15.2 (www.R-project.org) ${ }^{(21)}$. The linear mixed models were calculated using the lmerO function from the 'Ime4' package ${ }^{(22)}$, and the 'effects' package was used for plotting ${ }^{(23)}$.

\section{Results}

Complete information on $n-3$ and $n-6$ LC-PUFA concentrations or $n-6: n-3$ LC-PUFA ratio in CB serum glycerophospholipids and zBMI and LC-PUFA composition in serum glycerophospholipids at one or more follow-ups at 2, 6 or 10 years of age, as well as all covariables, was available for 388 children. To investigate the effect of selective dropout, all variables were tested for differences between participants and nonparticipants (Supplementary Table S1, available online), and none was found to differ significantly.

The basic characteristics of the study population are summarised in Table 1 . The percentage of LC-PUFA was higher in $\mathrm{CB}$ serum glycerophospholipids, although the $n-6: n-3$ LC-PUFA ratio remained stable. LC-PUFA concentrations increased slightly with age.

Tables 2-5 summarise the correlations between time of follow-up and zBMI, n-6 LC-PUFA concentrations, n-3 LC-PUFA concentrations and n-6:n-3 LC-PUFA ratio. zBMI measurements at 2, 6 and 10 years of age were positively correlated and reached statistical significance (Table 2). The lowest correlation was observed between 2 and 10 years of age (Pearson's correlation coefficient $r$ 0.2) and the highest between 6 and 10 years of age ( $r 0 \cdot 64)$. CB $n-6$ LC-PUFA concentrations were weakly, but significantly associated with $n-6$ LC-PUFA concentrations at 2 years $(r 0 \cdot 14)$ and 6 years $(r 0 \cdot 13)$ of age, but this result was no longer significant at 10 years (Table 3). Blood n-6 LC-PUFA concentrations at 2, 6 and 10 years of age were moderately correlated $(r \quad 0.45-0.53)$. For $n$-3 LC-PUFA concentrations and $n-6: n-3$ LC-PUFA ratio, the correlation pattern was similar, although the correlation coefficients were smaller (Tables 4 and 5).

Results obtained from the linear mixed models for zBMI are given in Table 6 . The concentrations of $n-6$ LC-PUFA and $n-3$ LC-PUFA or the ratio of $n$-6:n-3 LC-PUFA in CB was not significantly associated with zBMI later in life. The interaction term between time of follow-up and $n-6: n-3$ LC-PUFA ratio in CB was significant $(P=0 \cdot 0415)$. Fig. 2 shows the interaction effect between time of follow-up and $n-6: n-3$ LC-PUFA ratio in CB: a negative effect of the $n-6: n-3$ LC-PUFA ratio in CB was observed at 2 years; no effect was observed at 6 years; a positive effect was observed at 10 years. The interaction effects between $n-6$ and $n$ - 3 LC-PUFA concentrations in CB and time of follow-up were not significant $(P=0.1764$ and $0 \cdot 1870$, respectively). The effect of CB $n-6$ LC-PUFA concentrations was in the same direction (Supplementary Fig. S1(a), available online) as the interaction effect of $n$-6:n-3 LC-PUFA ratio in $\mathrm{CB}$, whereas the effect of $\mathrm{CB} n-3$ LC-PUFA was in the opposite direction (Supplementary Fig. S2(a), available online).

However, when the time-varying variables from the same year of follow-up were considered (Table 6), n-6 LC-PUFA concentrations were found to be associated with zBMI ( $\beta=0.02, P<0.0429)$. The association for $n$-3 LC-PUFA concentrations or the $n$-6:n-3 LC-PUFA ratio was not significant ( $\beta=-0 \cdot 18, P=0 \cdot 2813$, and $\beta=0 \cdot 28, P=0 \cdot 999$, respectively). Additionally, there was a significant interaction between time of follow-up and $n-6$ and $n$-3 LC-PUFA concentrations at the time of follow-up ( $P=0.0009$ and 0.0028 , respectively), but not the $n-6: n-3$ LC-PUFA ratio at the time of follow-up ( $P=0 \cdot 1356$; Supplementary Fig. S3, available online).

Supplementary Figs. S1(b) and S2(b) (available online) show the interaction effect between time of follow-up and $n-6$ and $n-3$ LC-PUFA concentrations at the time of followup, respectively. The concentrations of $n-6$ LC-PUFA exhibited a modest effect on zBMI at 2 and 6 years of age, whereas there was a strong positive effect at 10 years, indicating that children with a higher zBMI do have higher n-6 LC-PUFA concentrations (Supplementary Fig. S1(b), available online). The concentrations of $n$-3 LC-PUFA had a negative effect at 2 years, no effect at 6 years and a positive effect at 10 years of age (Supplementary Fig. S2(b), available online).

These results were further confirmed by stratified analyses using linear regression for each follow-up (Supplementary Table S2, available online). The direction of effect was the same as that of the interaction terms in the linear mixed models, although not all effects reached significance due to a reduced sample size.

In an additional sensitivity analysis, the linear mixed models were restricted to children with complete information on zBMI and fatty acid measurements at all time points (Supplementary Table S3, available online). The effect estimates were in the same direction of effect, although significance was not reached probably due to a reduced sample size (seventyfour children).

Table 3. Pearson's correlation between time of follow-up and $n-6$ long-chain PUFA (LC-PUFA) concentrations $\dagger$

\begin{tabular}{lcccc}
\hline$n-6$ LC-PUFA & CB & 2 years & 6 years & 10 years \\
\hline CB & 1 & & & \\
2 years & $0 \cdot 14^{\star}$ & 1 & & \\
6 years & $0 \cdot 13^{\star}$ & $0.43^{\star \star *}$ & 1 & \\
10 years & 0 & $0.47^{\star \star *}$ & $0.51^{\star \star \star}$ & 1 \\
\hline
\end{tabular}

$\mathrm{CB}$, cord blood

${ }^{*} P<0.05$, ${ }^{* * *} P<0.001$.

† Measured in serum glycerophospholipids. 
Table 4. Pearson's correlation between time of follow-up and $n-3$ long-chain PUFA (LC-PUFA) concentrations $†$

\begin{tabular}{lcccc}
\hline$n-3$ LC-PUFA & CB & 2 years & 6 years & 10 years \\
\hline $\mathrm{CB}$ & 1 & & & \\
2 years & $0.13^{\star}$ & 1 & & \\
6 years & 0.09 & $0.32^{\star \star \star}$ & 1 & \\
10 years & 0.1 & $0.27^{\star \star}$ & $0.35^{\star \star \star}$ & 1 \\
\hline
\end{tabular}

$\mathrm{CB}$, cord blood

${ }^{\star} P<0.05,{ }^{* *} P<0.01,{ }^{* \star *} P<0.001$

† Measured in serum glycerophospholipids.

\section{Discussion}

The present study investigated the association between $n-3$ and $n-6$ LC-PUFA concentrations and n-6:n-3 LC-PUFA ratio in $\mathrm{CB}$ serum glycerophospholipids and zBMI at 2, 6 and 10 years of age, after accounting for LC-PUFA percentages in serum glycerophospholipids using longitudinal data from the Munich LISAplus birth cohort.

The $n-6: n-3$ LC-PUFA ratio or $n-6$ and $n$-3 LC-PUFA concentrations in $\mathrm{CB}$ serum phospholipids were not consistently associated with zBMI. However, there was a significant interaction between $n$ - $6: n-3$ LC-PUFA ratio in CB and time of follow-up with respect to zBMI. A negative effect was observed at 2 years, no effect was observed at 6 years and a positive effect was observed at 10 years of age. The effect of $n-6$ LC-PUFA concentrations was in the same direction and that of $n$-3 LC-PUFA concentrations was in the opposite direction, but both were not significant.

When variables collected at the same time point were entered into the models, a higher zBMI was found to be associated with higher $n-6$ LC-PUFA percentages in serum glycerophospholipids, whereas the interaction effect between $n$-6 LC-PUFA concentrations and time of follow-up indicated that this effect was strongest at 10 years of age. The interaction term between $n-3$ LC-PUFA concentrations and time of follow-up suggests a positive effect on zBMI at 10 years, but a negative effect at 2 years of age.

Cross-sectional studies suggest that obese subjects have lower concentrations of $n$-3 LC-PUFA ${ }^{(24,25)}$. Therefore, studies have mainly focused on the supplementation of pregnant or breast-feeding women to improve the $n$ - 3 LC-PUFA supply to the offspring ${ }^{(13,26-31)}$. These interventional studies have led to inconclusive results. Courville et $a l^{(26)}$ reported lower ponderal indices at birth in an intervention group ( $n$ 22) receiving a DHA-containing functional food containing $300 \mathrm{mg}$ of DHA from mid-pregnancy to delivery compared with those in the control group ( $n$ 25) consuming a placebo bar containing maize oil. Another study carried out by Asserhøj et al. ${ }^{(27)}$ allocated women to high-fish intake ( $n$ 53) or low-fish intake ( $n$ 122) groups, and the women in the low-intake group were further allocated to receive fish-oil supplements containing $0.6 \mathrm{~g} \mathrm{EPA} / \mathrm{d}$ and $0.8 \mathrm{~g} \mathrm{DHA} / \mathrm{d}(n$ 62), or a control group supplemented with olive oil ( $n$ 60) from delivery to 4 months of lactation. The authors found a higher BMI in the supplementation group at $2 \cdot 5$ years, but this effect was no longer apparent at 7 years of age ${ }^{(27)}$. A supplementation study in
144 mothers randomised to receive one of three vitaminmineral supplements, of which one was supplying additional $200 \mathrm{mg} \mathrm{DHA} / \mathrm{d}$, from mid-pregnancy to 3 months conducted by Bergmann et al. ${ }^{(29)}$ found lower BMI and weight values in the infants allocated to the intervention group at 21 months of age, but there was no longer a difference between the intervention and control groups at 6 years of age ${ }^{(30)}$. Rosenfeld et al. ${ }^{(28)}$ conducted a meta-analysis based on individual patient data from four randomised clinical trials of postnatal supplementation with formula milk with and without LC-PUFA (DHA and AA). There was no difference in weight, length, head circumference or BMI between 442 supplemented children and 459 control children at 18 months ${ }^{(28)}$. The INFAT Study, a clinical trial, investigated the effect of detailed nutritional counselling and supplementation with $1200 \mathrm{mg}$ DHA/d from 15th week of gestation to 4 months after delivery on the offspring's body composition assessed at several time points during the first year of life. The control group received brief semi-structured counselling on a healthy diet. Although there was a significant increase in $n-3$ LC-PUFA concentrations and a decrease in AA concentrations in maternal blood in the intervention group, there were no significant differences between the intervention and control groups with regard to any of the investigated growth and body composition parameters ${ }^{(13)}$. Maternal AA and n-6 LC-PUFA concentrations were inversely associated with BMI at 1 year of age, but not with fat mass ${ }^{(31)}$. A randomised controlled intervention study carried out by Andersen et $a l .{ }^{(32)}$ investigated the effect of fish oil $(1.6 \mathrm{~g} \mathrm{EPA}+\mathrm{DHA} / \mathrm{d})$ $v$. sunflower oil in 133 infants from 9 to 18 months of age. There was no significant difference in any of the anthropometric measures related to fat mass, but the infants in the sunflower group had a lower skinfold ratio (tricipital:subscapular) at 18 months of age.

Donahue et $a l^{(33)}$ reported results from an observational study that investigated associations between childhood obesity at 3 years of age and maternal dietary intake and maternal plasma fatty acid composition during pregnancy, as well as CB PUFA concentrations. Obesity (BMI $\geq 95$ th percentile) and the sum of skinfold thicknesses were negatively associated with CB $n$-3 LC-PUFA concentrations and positively associated with the $n-6: n-3$ LC-PUFA ratio. zBMI were associated with maternal plasma total $n$ - 6 LC-PUFA concentrations but not with CB PUFA concentrations.

Table 5. Pearson's correlation between time of follow-up and $n-6: n-3$ long-chain PUFA (LC-PUFA) ratioł

\begin{tabular}{lcccc}
\hline$n-6: n-3$ LC-PUFA ratio & $\mathrm{CB}$ & 2 years & 6 years & 10 years \\
\hline $\mathrm{CB}$ & 1 & & & \\
2 years & $0.15^{\star}$ & 1 & & \\
6 years & $0.11 \dagger$ & $0.39^{\star \star *}$ & 1 & \\
10 years & $0.11 \dagger$ & $0.22^{* \star}$ & $0.46^{\star \star *}$ & 1 \\
\hline
\end{tabular}

$\mathrm{CB}$, cord blood.

${ }^{\star} P<0.05,{ }^{* \star} P<0.01,{ }^{* \star *} P<0.001$.

$\dagger P<0 \cdot 1$.

$\ddagger$ Measured in serum glycerophospholipids. 
Table 6. Results obtained from the linear mixed models for BMl $z$-scores (zBMI) $v$. $n$ - 6 long-chain PUFA (LC-PUFA) concentrations, $n$-3 LC-PUFA concentrations and $n-6: n-3$ LC-PUFA ratio in cord blood (CB) (PUFA $A_{C B}$ ) and at follow-up (PUFA $\left.A_{F}\right)$ ( $\beta$ values with their standard errors)

\begin{tabular}{|c|c|c|c|c|c|c|c|c|c|}
\hline & \multicolumn{3}{|c|}{$\begin{array}{l}n-6 \text { LC-PUFA } † \\
(n \text { 385) }\end{array}$} & \multicolumn{3}{|c|}{$\begin{array}{l}n-3 \text { LC-PUFA }+ \\
(n \text { 384) }\end{array}$} & \multicolumn{3}{|c|}{$\begin{array}{c}n-6: n-3 \text { LC-PUFA ratio } † \\
(n \text { 387) }\end{array}$} \\
\hline & $\beta \ddagger$ & SE & $P$ & $\beta \ddagger$ & SE & $P$ & $\beta \ddagger$ & SE & $P$ \\
\hline PUFA $_{C B}$ & -0.04 & 0.04 & 0.8050 & 0.02 & 0.04 & 0.7639 & -0.11 & 0.09 & $0 \cdot 8017$ \\
\hline PUFA $_{F} \S$ & 0.02 & 0.04 & $0.0429^{\star}$ & -0.18 & 0.08 & 0.2813 & 0.28 & $0 \cdot 13$ & 0.9990 \\
\hline Time $_{6 \text { years }} \times$ PUFA $_{C B} \|$ & 0.04 & 0.05 & 0.1764 & -0.03 & 0.04 & 0.1870 & 0.14 & $0 \cdot 10$ & $0.0415^{\star}$ \\
\hline Time $_{10 \text { years }} \times$ PUFA $_{C B} \|$ & 0.07 & 0.05 & & -0.09 & 0.04 & & 0.27 & $0 \cdot 10$ & \\
\hline Time $_{6 \text { years }} \times$ PUFA $_{F} \S \|$ & -0.05 & 0.05 & $0.0009^{*}$ & 0.17 & 0.09 & $0.0028^{\star}$ & -0.32 & 0.15 & 0.1356 \\
\hline Time $_{10 \text { years }} \times$ PUFA $_{F} \S \|$ & 0.08 & 0.05 & & 0.32 & 0.09 & & -0.41 & $0 \cdot 15$ & \\
\hline
\end{tabular}

${ }^{\star} P<0.05$.

† Measured in serum glycerophospholipids.

$\ddagger$ Estimates were adjusted for birth weight, maternal BMI before pregnancy, exclusive breast-feeding for $>4$ months, time of follow-up (2, 6 or 10 years; reference: 2 years) and age at follow-up, respectively.

$\S$ Combined estimate for the LC-PUFA composition at 2, 6 and 10 years of age.

II Interaction terms between time of follow-up and PUFA concentrations in CB and PUFA concentrations at follow-up.

Another observational study carried out by Moon et al. ${ }^{(34)}$ investigated the association between maternal plasma PUFA concentrations and fat mass and lean mass at 4 and 6 years of age. Higher maternal plasma $n-6$ PUFA concentrations were related to higher offspring fat mass at 4 years and 6 years of age, but not with lean mass. There was no effect of maternal $n-3$ PUFA concentrations. Moon et al. ${ }^{(34)}$ suggested that reducing $n-6$ LC-PUFA concentrations may be a more effective method to reduce BMI than increasing $n-3$ LC-PUFA concentrations.

In summary, observational and interventional studies have yielded an inconsistent picture of the association between LC-PUFA concentrations in early life and BMI. Most of these studies suffer from having a small sample size, in particular, the interventional studies, and fatty acid composition in blood was usually measured only once. No study has yet attempted to longitudinally model the association between repeated LC-PUFA concentrations and BMI.
The present results indicate a time-dependent effect of $\mathrm{CB}$ serum glycerophospholipid LC-PUFA concentrations on BMI. The ratio of $n-6: n-3$ LC-PUFA in CB had a negative effect at 2 years, no effect at 6 years and a positive effect at 10 years of age. Although non-significant, the effect of $n$ - 6 LC-PUFA concentrations in $\mathrm{CB}$ was in the same direction and the effect of $n$-3 LC-PUFA concentrations in CB serum glycerophospholipids was in the opposite direction. Considering only the results obtained at 10 years of age, the results are in line with the hypothesis introduced by Ailhaud \& Guesnet ${ }^{(5)}$. However, consequently, the results reported herein for 2 years of age go against this hypothesis. At 6 years of age, which is approximately the time of adiposity rebound, we found no effect. During childhood, BMI increases from birth up to the adiposity peak, which occurs at approximately 8-9 months of age. Afterwards, BMI decreases until the point of adiposity rebound, which occurs within the age range of 5-7 years.

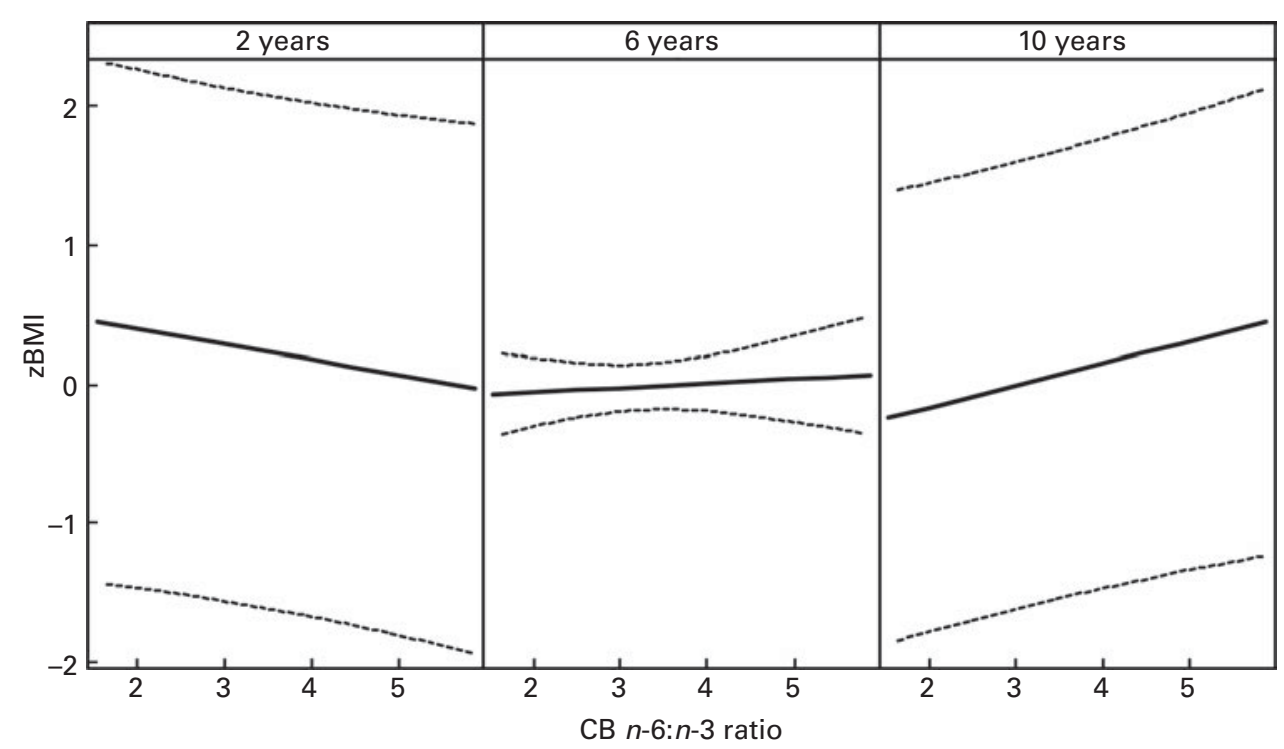

Fig. 2. Interaction effect between $n-6: n-3$ ratio in cord blood (CB) serum glycerophospholipids and time of follow-up (2, 6 and 10 years) on BMI $z$-scores (zBMI). The — represents the effect; the ..... represent the corresponding $95 \% \mathrm{Cl}$. $P$ value of the interaction=0.0415. 
Usually, an early adiposity rebound predicts a higher BMI later in life ${ }^{(35)}$.

The main limitation of the present study is that detailed measurements of body composition and fat mass were not available. These data would have provided more detailed information on weight status rather than BMI data. However, it has been shown that the percentage of body fat and BMI have similar patterns over time ${ }^{(36)}$

The longitudinal study design allows the effect of CB serum glycerophospholipid LC-PUFA concentrations on BMI at several time points up to 10 years of age to be modelled. Data on LC-PUFA concentrations were available at birth and 2, 6 and 10 years of age. CB fatty acid composition represents the fatty acid supply to the fetus before birth. The models were adjusted for the fatty acid composition in blood at each follow-up to rule out confounding by lifestyle or dietary factors, which may be reflected in fatty acid concentrations in blood. As we were interested in blood fatty acids, we did not adjust for potential dietary sources. In addition, data on fish intake were not collected at each follow-up.

The longitudinal approach used increased the statistical power of the study, as the maximal number of observations available was included in the models.

Another limitation of the present study is the potential bias associated with non-random loss to follow-up. However, a sensitivity analysis restricted to children with complete data for all the time points of follow-up yielded similar results, although not significant due to the limited sample size (Supplementary Table S3, available online), and there were no significant differences in the investigated variables between participants and non-participants (Supplementary Table S1, available online).

More longitudinal studies are needed to assess the longterm effect of CB LC-PUFA concentrations on body composition and weight development. Only with additional studies will we be able to develop well-justified dietary recommendations during pregnancy.

In summary, BMI up to 10 years of age may be influenced by the ratio of $n$ - $6: n-3$ LC-PUFA in CB serum glycerophospholipids. However, this effect appears to vary with age. The present results thereby highlight the importance of considering age when examining associations between fatty acid concentrations and BMI.

\section{Supplementary material}

To view supplementary material for this article, please visit http://dx.doi.org/10.1017/S0007114514000105

\section{Acknowledgements}

The authors thank all the families who participated in the LISAplus Study. The authors also thank all the members of the LISAplus Study group for their excellent work. They also acknowledge Larissa Piniek-Tzimourtas (Division of Metabolic and Nutritional Medicine, University of Munich Medical Center, Dr von Hauner Children's Hospital, Munich, Germany) for her excellent contribution to the measurements of fatty acid composition. The LISAplus Study group consists of the following: Helmholtz Zentrum München, German Research Center for Environmental Health, Institute of Epidemiology I, Munich (J. Heinrich, H. E. Wichmann, S. Sausenthaler, C. M. Chen and M. Schnappinger); Department of Pediatrics, Municipal Hospital 'St Georg', Leipzig (M. Borte and U. Diez); Marien-Hospital Wesel, Department of Pediatrics, Wesel (A. von Berg, C. Beckmann, and I. Groß); Pediatric Practice, Bad Honnef (B. Schaaf); Helmholtz Centre for Environmental Research-UFZ, Department of Environmental Immunology/Core Facility Studies, Leipzig (I. Lehmann, M. Bauer, C. Gräbsch, S. Röder and M. Schilde); University of Leipzig, Institute of Hygiene and Environmental Medicine, Leipzig (O. Herbarth, C. Dick and J. Magnus); IUF-Institut für Umweltmedizinische Forschung, Düsseldorf (U. Krämer, E. Link and C. Cramer); Technical University Munich, Department of Pediatrics, Munich (C. P. Bauer and U. Hoffmann); ZAUM-Center for Allergy and Environment, Technical University, Munich (H. Behrendt, J. Grosch and F. Martin).

The present study was supported by grant no. 01 EG 9732, 01 EG 9705/2 and 01 EE 9401-4 from the Federal Ministry for Education, Science, Research and Technology; FKZ 20462296 from the Federal Ministry of Environment; Ludwig Maximilians University's innovative research priority project MC-Health; the Kompetenznetz Adipositas (Competence Network Obesity) funded by the Federal Ministry of Education and Research (FKZ: 01GI1121A); the Commission of the European Communities; and a specific RTD programme NUTRIMENTHE (FP7-212652). B. K. is a recipient of the Freedom to Discover Award of the Bristol-Myers Squibb Foundation, New York, NY. None of the funders had a role in the design and analysis of the study or in the writing of this article.

The authors' contributions are as follows: B. K. and J. H. designed the study; H. D. carried out fatty acid measurements; E. T. and M. S. analysed the data. All authors were involved in interpretation of results, writing of the paper and final approval of the submitted and published version.

None of the authors has any conflicts of interest to declare.

\section{References}

1. Reilly JJ \& Kelly J (2011) Long-term impact of overweight and obesity in childhood and adolescence on morbidity and premature mortality in adulthood: systematic review. Int J Obes (Lond) 35, 891-898.

2. Franks PW, Hanson RL, Knowler WC, et al. (2010) Childhood obesity, other cardiovascular risk factors, and premature death. $N$ Engl J Med 362, 485-493.

3. Han JC, Lawlor DA \& Kimm S (2010) Childhood obesity. Lancet 375, 1737-1748.

4. Muhlhausler BS \& Ailhaud GP (2013) Omega-6 polyunsaturated fatty acids and the early origins of obesity. Curr Opin Endocrinol Diabetes Obes 20, 56-61.

5. Ailhaud G \& Guesnet P (2004) Fatty acid composition of fats is an early determinant of childhood obesity: a short review and an opinion. Obes Rev 5, 21-26.

6. Haggarty P (2002) Placental regulation of fatty acid delivery and its effect on fetal growth - a review. Placenta 23, S28-S38.

7. Larqué E, Demmelmair H, Gil-Sánchez A, et al. (2011) Placental transfer of fatty acids and fetal implications. Am J Clin Nutr 94, Suppl. 6, 1908S-1913S. 
8. Berghaus TM, Demmelmair H \& Koletzko B (2000) Essential fatty acids and their long-chain polyunsaturated metabolites in maternal and cord plasma triglycerides during late gestation. Biol Neonate 77, 96-100.

9. Donahue SMA, Rifas-Shiman SL, Olsen SF, et al. (2009) Associations of maternal prenatal dietary intake of $n-3$ and $n-6$ fatty acids with maternal and umbilical cord blood levels. Prostaglandins Leukot Essent Fatty Acids 80, 289-296.

10. Ailhaud G, Guesnet P \& Cunnane SC (2008) An emerging risk factor for obesity: does disequilibrium of polyunsaturated fatty acid metabolism contribute to excessive adipose tissue development? Br J Nutr 100, 461-470.

11. Delgado-Noguera MF, Calvache JA \& Bonfill Cosp (2010) Supplementation with long chain polyunsaturated fatty acids (LCPUFA) to breastfeeding mothers for improving child growth and development. The Cochrane Database of Systematic Reviews, CD007901.

12. Martínez-Victoria E \& Yago MD (2012) Omega 3 polyunsaturated fatty acids and body weight. Br J Nutr 107, Suppl. 2, S107-S116.

13. Hauner H, Much D, Vollhardt C, et al. (2012) Effect of reducing the $n-6: n-3$ long-chain PUFA ratio during pregnancy and lactation on infant adipose tissue growth within the first year of life: an open-label randomized controlled trial. Am J Clin Nutr 95, 383-394.

14. Hauner H, Vollhardt C, Schneider KTM, et al. (2009) The impact of nutritional fatty acids during pregnancy and lactation on early human adipose tissue development. Rationale and design of the INFAT study. Ann Nutr Metab 54, 97-103.

15. Heinrich J, Bolte G, Hölscher B, et al. (2002) Allergens and endotoxin on mothers' mattresses and total immunoglobulin $\mathrm{E}$ in cord blood of neonates. Eur Respir J 20, 617-623.

16. Butte NF, Garza C \& de Onis M (2007) Evaluation of the feasibility of international growth standards for school-aged children and adolescents. J Nutr 137, 153-157.

17. Glaser C, Demmelmair H \& Koletzko B (2010) High-throughput analysis of fatty acid composition of plasma glycerophospholipids. J Lipid Res 51, 216-221.

18. Kohlboeck G, Glaser C, Tiesler C, et al. (2011) Effect of fatty acid status in cord blood serum on children's behavioral difficulties at $10 \mathrm{y}$ of age: results from the LISAplus Study. Am J Clin Nutr 94, 1592-1599.

19. Glaser C, Rzehak P, Demmelmair H, et al. (2011) Influence of FADS polymorphisms on tracking of serum glycerophospholipid fatty acid concentrations and percentage composition in children. PLoS One 6, e21933.

20. Cnaan A, Laird NM \& Slasor P (1997) Using the general linear mixed model to analyse unbalanced repeated measures and longitudinal data. Stat Med 16, 2349-2380.

21. R Development Core Team (2012) $R$ : A Language and Environment for Statistical Computing. Vienna: R Foundation for Statistical Computing.

22. Bates D, Maechler M \& Bolker B (2011) lme4: linear mixedeffects models using $\mathrm{S} 4$ classes. $\mathrm{R}$ package version $0.999375-42$.
23. Fox J (2003) Effect displays in $\mathrm{R}$ for generalised linear models. J Stat Softw 8, 1-27.

24. Micallef M, Munro I, Phang M, et al. (2009) Plasma n-3 polyunsaturated fatty acids are negatively associated with obesity. Br J Nutr 102, 1370-1374.

25. Karlsson M, Mårild S, Brandberg J, et al. (2006) Serum phospholipid fatty acids, adipose tissue, and metabolic markers in obese adolescents. Obesity (Silver Spring) 14, 1931-1939.

26. Courville AB, Harel O \& Lammi-Keefe CJ (2011) Consumption of a DHA-containing functional food during pregnancy is associated with lower infant ponderal index and cord plasma insulin concentration. Br J Nutr 106, 208-212.

27. Asserhøj M, Nehammer S, Matthiessen J, et al. (2009) Maternal fish oil supplementation during lactation may adversely affect long-term blood pressure, energy intake, and physical activity of 7-year-old boys. J Nutr 139, 298-304.

28. Rosenfeld E, Beyerlein A, Hadders-Algra M, et al. (2009) IPD meta-analysis shows no effect of LC-PUFA supplementation on infant growth at 18 months. Acta Paediatr 98, 91-97.

29. Bergmann RL, Bergmann KE, Haschke-Becher $\mathrm{E}$, et al. (2007) Does maternal docosahexaenoic acid supplementation during pregnancy and lactation lower BMI in late infancy? J Perinat Med 35, 295-300.

30. Bergmann RL, Bergmann KE, Richter R, et al. (2012) Does docosahexaenoic acid (DHA) status in pregnancy have any impact on postnatal growth? Six-year follow-up of a prospective randomized double-blind monocenter study on low-dose DHA supplements. J Perinat Med 40, 677-684.

31. Much D, Brunner S, Vollhardt C, et al. (2013) Effect of dietary intervention to reduce the $n-6 / n-3$ fatty acid ratio on maternal and fetal fatty acid profile and its relation to offspring growth and body composition at 1 year of age. Eur J Clin Nutr 67, 282-288.

32. Andersen AD, Michaelsen KF, Hellgren LI, et al. (2011) A randomized controlled intervention with fish oil versus sunflower oil from 9 to 18 months of age: exploring changes in growth and skinfold thicknesses. Pediatr Res 70, 368-374.

33. Donahue SMA, Rifas-Shiman SL, Gold DR, et al. (2011) Prenatal fatty acid status and child adiposity at age $3 \mathrm{y}$ : results from a US pregnancy cohort. Am J Clin Nutr 93, $780-788$

34. Moon RJ, Harvey NC, Robinson SM, et al. (2013) Maternal plasma polyunsaturated fatty acid status in late pregnancy is associated with offspring body composition in childhood. J Clin Endocrinol Metab 98, 299-307.

35. Williams SM \& Goulding A (2009) Patterns of growth associated with the timing of adiposity rebound. Obesity (Silver Spring) 17, 335-341.

36. Plachta-Danielzik S, Gehrke MI, Kehden B, et al. (2012) Body fat percentiles for German children and adolescents. Obes Facts 5, 77-90. 